\title{
Cristaux liquides : l'univers métastable des monopôles nématiques
}

Pawel Pieranski (pawel.pieranski@u-psud.fr)

Prix Félix Robin 2015 de la Société Française de Physique

Laboratoire de physique des solides, Bât. 510, Université Paris-Sud, 91405 0rsay

Les cristaux liquides permettent d'illustrer facilement le concept universel de défauts

topologiques, incontournable en physique de la matière condensée.

\section{Nous décrivons ici une} expérience permettant de générer la texture métastable dite pseudo-planaire des cristaux liquides nématiques : cette texture est particulièrement adaptée à l'étude des défauts

ponctuels de ceux-ci.

On peut les y créer par paires monopôle-antimonopôle

et étudier leurs collisions

et leur section efficace

d'annihilation.

Cette texture est aussi douée de cunéitropisme, c'est-à-dire de la faculté de s'orienter dans un gradient d'épaisseur, dont une des conséquences est la formation de parois qui ont toutes les caractéristiques de solitons.

\section{Défauts topologiques}

Aujourd'hui, les concepts de défauts topologiques linéaires (dislocations, disinclinaisons, vortex supraconducteurs ou superfluides) ou ponctuels (monopôles électriques, magnétiques ou nématiques) [1] sont incontournables en physique de la matière condensée. S'agissant de concepts universels, il suffit de les illustrer par un exemple dans un de ces systèmes pour donner une certaine idée de ce qu'ils peuvent être dans tous les autres. Par la facilité de leur utilisation, les cristaux liquides se prêtent très bien à cet exercice, comme nous l'avions montré dans un livre rédigé en commun avec Patrick Oswald [2]. $\mathrm{Si}$ les défauts linéaires - disinclinaisons (encadré 1 et fig. E2a) - sont très faciles à produire dans un échantillon de cristal liquide nématique, la génération des défauts ponctuels [3] - appelés monopôles (fig. E2b) - demande plus d'efforts.

Nous allons montrer ici comment créer un " univers " où on peut faire naitre, évoluer, collisionner et annihiler des paires monopôle-antimonopôle. Cet univers est la texture nématique métastable, dite pseudo-planaire, étudiée auparavant par J.M. Gilli et al. [4]. Pour commencer nous allons montrer comment, contrairement aux idées reçues, on peut rendre sa durée de vie pratiquement infinie.

\section{Cellule nématique d'épaisseur variable}

Notre expérience, représentée sur la figure 1 (p. 8), a été réalisée avec une cellule nématique formée d'une couche de cristal liquide confinée entre une lentille sphérique et une lame de verre plane ; cette cellule est elle-même placée entre un polariseur $\mathrm{P}$ et un analyseur A croisés. La distance de séparation $\mathrm{h}$ est réglable typiquement entre 0 et quelques $\mathrm{mm}$, et elle augmente à cause de la convexité de la lentille lorsqu'on s'écarte de l'axe de la cellule. Les surfaces de verre ont été traitées (au jaune d'œuf cuit) pour orienter les molécules du nématique dans la direction $\mathrm{z}$ perpendiculaire aux surfaces. En volume, l'orientation commune des molécules, repérée localement par le vecteur unitaire $\mathbf{n}(\mathrm{z})$, doit alors se raccorder avec celle des molécules sur les surfaces. Dans l'état d'énergie libre minimale, l'orientation des molécules du cristal liquide est uniforme et parallèle à l'axe z. Cet état porte le nom de texture homéotrope, selon la terminologie introduite en 1920 par Georges Friedel qui a, par ailleurs, inventé l'appellation de "nématique " (voir encadré 2, p. 9).

Tout expérimentateur ayant préparé des cellules avec cette texture homéotrope sait qu'elle n'est pas obtenue immédiatement après le remplissage de la cellule. L'écoulement du cristal liquide lors du remplissage tend en effet à orienter les molécules en favorisant plutôt une autre structure appelée pseudo-planaire (figures $1 \mathrm{a}$ et $1 \mathrm{~d}$ ) ; cette structure est aussi favorisée par la présence à la périphérie du cristal liquide d'un ménisque d'interface auquel les molécules sont également perpendiculaires. À l'instar de la texture homéotrope, la texture pseudo-planaire satisfait les conditions aux limites (n perpendiculaire aux plaques de verre) mais, en volume, la direction commune des molécules $\mathbf{n}(z)$ tourne de $\pi$ entre les deux plaques (figures $1 \mathrm{a}$ et $1 \mathrm{~d}$ ). 


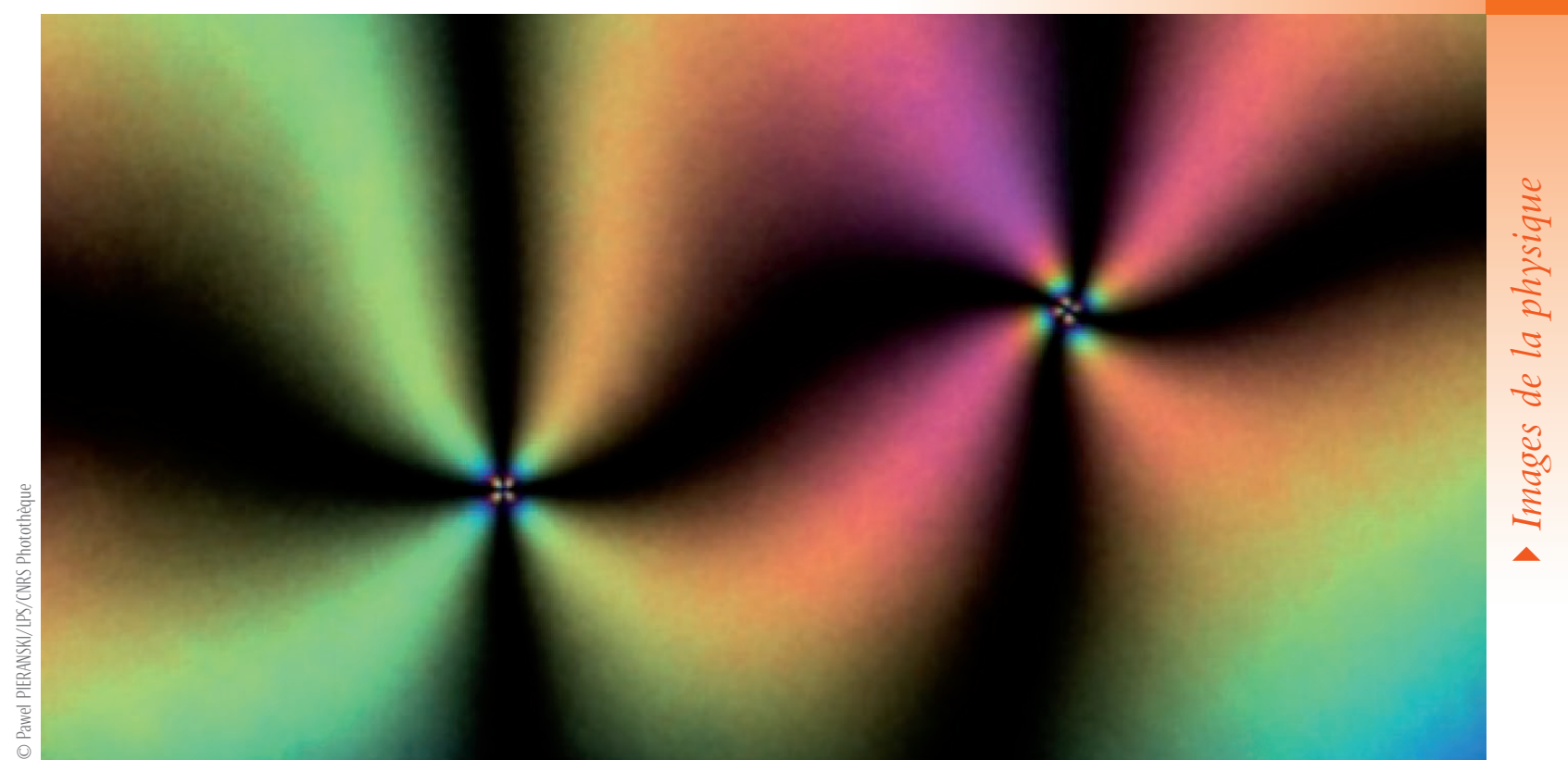

Défauts topologiques d'un cristal liquide : singularités ponctuelles du « champ du directeur » situées à l'interface nématique/isotrope (photo prise entre polariseurs croisés).

\section{Les cristaux liquides nématiques et leurs propriétés}

encadré 1

Un nématique est un type particulier de cristal liquide, aux molécules allongées et généralement organiques. Au lieu d'être aléatoires comme dans un liquide isotrope, les orientations de ces molécules sont majoritairement parallèles à un vecteur unitaire $\mathbf{n}$ appelé directeur (fig. E1). Par contre, d'une part les positions des centres de gravité de ces molécules sont aléatoires au lieu d'être ordonnées comme dans un cristal et, d'autre part, elles peuvent s'écouler comme dans un liquide ordinaire. La direction $\mathbf{n}$ ou $\mathbf{n} \mathbf{n} \mathbf{d u}$ directeur est sans importance, car la phase nématique n'a pas de polarisation spontanée. Dans un nématique sans défauts, l'orientation du directeur $\mathbf{n}$ est uniforme ou varie lentement dans l'espace. Les échantillons nématiques « réels » présentent fréquemment des défauts sous forme de lignes (par exemple les disinclinaisons de la figure E2a) ou de points (par exemple les monopôles de la figure E2b), autour desquels l'orientation du vecteur $\mathbf{n}$ varie rapidement; le nom de nématique vient d'ailleurs des défauts linéaires qui apparaissent au microscope comme des fils (nêma en grec), flottant dans le liquide.

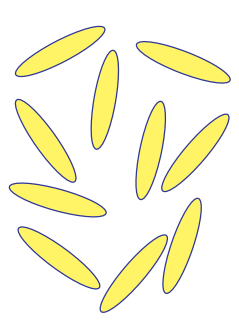

Phase isotrope

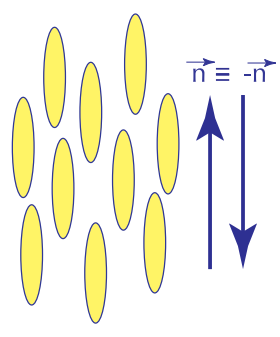

Phase nématique

E1. Orientation des molécules dans la phase isotrope et dans la phase nématique.

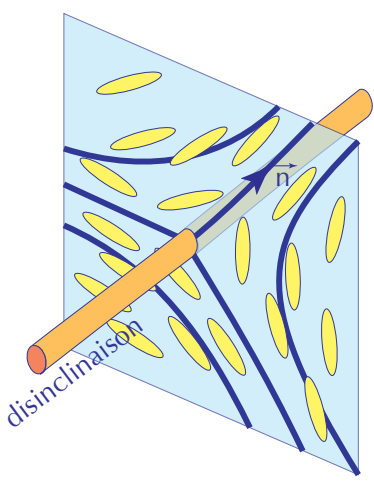

$\mathrm{a}$

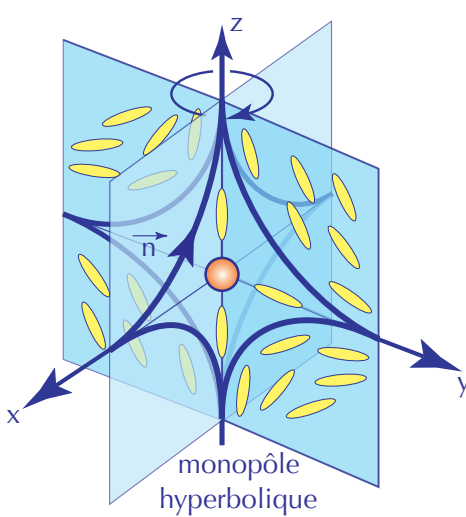

$\mathrm{b}$
E2. Défauts topologiques de la phase nématique.

a) Défaut linéaire appelé disinclinaison. Le schéma représente les orientations des molécules dans un plan orthogonal à la disinclinaison. Les lignes de champ (en bleu) sont, par définition, tangentes en tout point au directeur $\mathbf{n}$ et, ainsi, localement parallèles aux molécules. La disinclinaison est donc une singularité linéaire du champ du directeur.

b) Défaut ponctuel appelé monopôle hyperbolique, car les lignes du champ du directeur $\mathbf{n}$ dessinées en bleu ont la forme d'hyperboles dont l'une des asymptotes est parallèle à l'axe z. L'autre asymptote est radiale et perpendiculaire à l'axe z. Ce monopôle hyperbolique a une symétrie de révolution autour de l'axe z. On le retrouve sur les schémas d et e de la figure 1. Les monopôles nématiques peuvent avoir d'autres structures, comme nous en verrons un exemple dans la figure $5 \mathrm{~d}$.

Les cristaux liquides nématiques sont généralement biréfringents, avec un indice optique différent suivant l'orientation de la polarisation de la lumière. Dans la texture homéotrope au centre des figures 1a et $1 \mathrm{~b}$ où le vecteur $\mathbf{n}$ est parallèle à $z$, un faisceau lumineux parallèle à l'axe z se propage comme dans un milieu isotrope car sa polarisation est perpendiculaire à n. Si la lumière incidente est polarisée rectilignement et si l'axe de l'analyseur A est perpendiculaire à celui du polariseur P (polariseurs croisés), la zone correspondante à la sortie de l'analyseur A est noire (fig. 1c). Dans la zone pseudo-planaire, au contraire, le directeur $\mathrm{n}$ est incliné par rapport à la direction z de propagation de la lumière : la lumière à la sortie de l'échantillon n'est généralement plus arrêtée par l'analyseur, car sa polarisation est elliptique (elle combine deux composantes propres de polarisation correspondant à des indices optiques différents). 


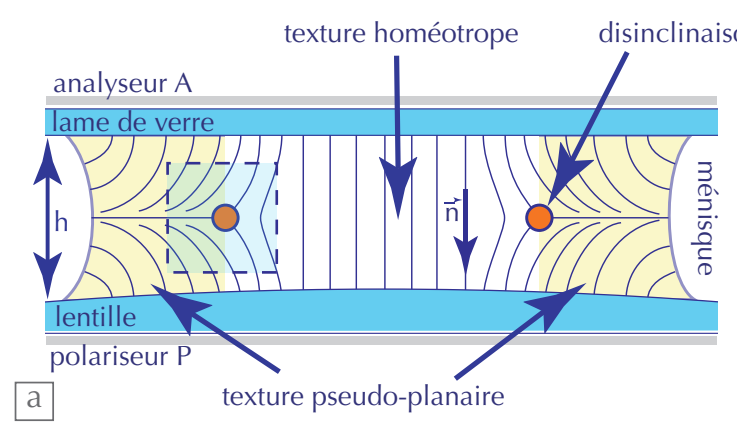

$\mathrm{b}$
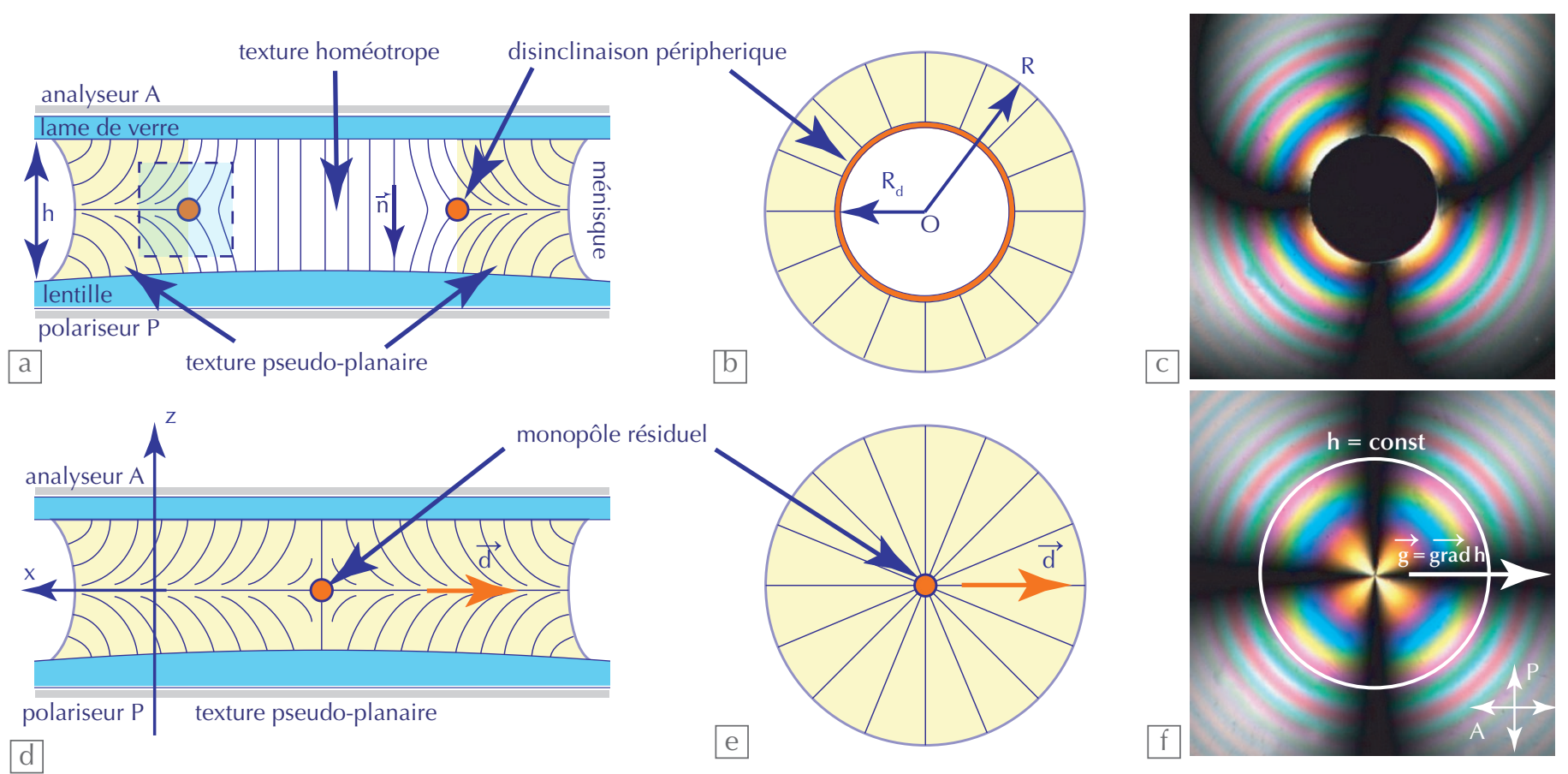

1. Compétition entre les textures homéotrope et pseudo-planaire dans un échantillon de nématique confiné entre une lentille et une lame plane, avec ancrage homéotrope (perpendiculaire aux parois) des molécules.

(a, b, d, e) Schémas dans le plan vertical x0z (a, d) et horizontal x0y (b, e) de la structure du cristal liquide. En bleu : lignes de champ du directeur $\mathbf{n}$ (le champ à l'intérieur du petit rectangle bleu dans (a) est le même que dans la figure EZa). Le vecteur $\mathbf{d}$ est la composante azimutale (i.e. dans le plan (x,y)) du directeur $\mathbf{n}$.

(c, f) Vues de dessus entre polariseurs croisés (A et P) et avec un éclairage suivant l'axe $0 z$ des structures représentées respectivement dans (a, b) et (d, e).

(a, b, c) Coexistence d'une texture homéotrope (en blanc dans (a, b) et disque noir dans (c)) et d'une texture pseudo-planaire (en jaune dans (a, b), franges colorées et croix noire dans (c)), séparées par une ligne de disinclinaison (en orange).

(d, e, f) Structure pseudo-planaire (mêmes présentations que dans (a, b, c)) avec un monopôle résiduel au centre (en orange). Dans l'image f, les flèches en bas à droite représentent les orientations des polarisations pour le polariseur $P$ et l'analyseur $A$.

$>>$

Dans la texture pseudo-planaire, les molécules du cristal liquide ne sont pas parallèles entre elles partout comme elles le sont dans la texture homéotrope, ce qui fait augmenter l'énergie libre du système. L'écart par rapport à l'orientation uniforme d'énergie minimale s'exprime au premier ordre par la « vitesse spatiale " de rotation $\mathrm{d} \theta / \mathrm{dz} \approx \pi / \mathrm{h}$, où $\theta$ est l'angle du vecteur $\mathbf{n}$ avec l'axe $z$.

L'accroissement de l'énergie libre par unité de volume qui en résulte s'écrit $\mathrm{f}_{\text {él }}=(\mathrm{K} / 2)(\pi / \mathrm{h})^{2}$ ou bien, après l'intégration sur l'épaisseur h, $\mathrm{F}_{\text {él }}=(\mathrm{K} / 2)\left(\pi^{2} / \mathrm{h}\right)$ par unité de surface ( $\mathrm{K}$ est une constante d'élasticité, dite d'Oseen-Frank).

\section{Surprises, ou « le diable est dans les détails »}

Ayant préparé quelques dizaines de cellules homéotropes tout au long de ma carrière, j'étais convaincu que la texture pseudoplanaire, déformée, mise en compétition avec la texture homéotrope, homogène, "perd » irrémédiablement pour des raisons énergétiques et qu'il suffit d'attendre une demi-heure pour obtenir une belle texture homéotrope optiquement uniaxe.

Or, récemment, pendant une étude des disinclinaisons captives sur des fibres polymériques une surprise est survenue : dans un échantillon inhabituellement épais (2 $\mathrm{mm}$ au lieu de $0,2 \mathrm{~mm}$ ), la texture homéotrope a été éliminée par la texture pseudo-planaire.
L'explication de ce phénomène est simple et elle était connue de J.M. Gilli et al. [4]. Dans le bilan énergétique de la cellule où les deux textures coexistent (figures $1 \mathrm{a}, \mathrm{b}$ et c), il faut tenir compte non pas d'une mais de deux contributions. La première concerne les énergies de déformation élastique par unité de surface $\mathrm{F}_{\text {él }}$ dont nous venons de discuter : celle de la texture homéotrope homogène est nulle, tandis que celle de la texture pseudo-planaire est positive, $F_{\text {él }}=(K / 2)\left(\pi^{2} / h\right)$, et varie comme $1 /$ h. Dans la géométrie circulaire des figures $1 a-b$, cette énergie par unité de surface peut être aussi vue comme une force par unité de longueur qui agit sur la frontière entre les deux textures et tend à augmenter le rayon $\mathrm{R}_{\mathrm{d}}$ de la texture homéotrope. 
La deuxième contribution est celle de la tension $\mathrm{T}(\mathrm{h})$ de la disinclinaison. Elle crée une force de Laplace $T(h) / R_{d}$ centripète qui tend à diminuer le rayon $\mathrm{R}_{\mathrm{d}}$.

L'issue de la compétition entre les deux forces dépend de l'épaisseur $\mathrm{h}$ et, dans notre échantillon inhabituellement épais, la texture pseudo-planaire a éliminé la texture homéotrope en laissant cependant un vestige : le monopôle résiduel (figures $1 \mathrm{~d}$, e et $\mathrm{f}$ ) résultant de l'effondrement de la disinclinaison. Remarquons que la structure de ce monopôle est la même que celle du monopôle de la figure E1b. Nous allons en reparler à la fin de l'article.

Après cette première surprise est venue une seconde : l'étonnante robustesse de la texture pseudo-planaire ; en effet, on pourrait croire qu'en diminuant l'épaisseur de la cellule on permettrait à la texture homéotrope de redevenir championne comme d'accoutumée. Cependant, en s'imaginant cela on oublie que la texture homéotrope est absente de la cellule. Pour rendre possible sa nucléation, il faudrait réduire l'épaisseur h à moins d'un micromètre. En conséquence, en prenant garde de ne pas trop réduire l'épaisseur h de la cellule, nous avons pu soumettre la texture pseudoplanaire à quelques expériences qui ont révélé ses remarquables propriétés.

\section{Cunéitropisme}

La première de ces propriétés est le cunéitropisme, c'est-à-dire, selon l'étymologie (du latin cuneus $=$ coin) de ce néologisme (voir l'encadré 2), que l'orientation moyenne des molécules devient parallèle au gradient d'épaisseur. Nous allons montrer que c'est bien le cunéitropisme qui est à l'origine de la figure d'interférence visible sur la figure 1f, obtenue par observation de l'échantillon (selon l'axe z) placé entre un polariseur et un analyseur croisés. Pour simplifier la discussion, nous allons utiliser l'image de la figure $1 \mathrm{f}$ mais obtenue avec une lumière monochromatique.

Pour commencer, remarquons que, sur l'image de la figure 1f obtenue avec une lumière blanche, on distingue d'abord un système de franges circulaires colorées qu'on appelle isochromes. Sur la figure 2, obtenue avec une lumière monochromatique, les isochromes sont aussi circulaires mais noires. L'optique des milieux anisotropes nous enseigne en effet qu'on a une différence $\Delta \mathrm{n}$ entre les indices optiques pour la propagation des rayons lumineux de polarisation parallèle et perpendiculaire à la projection $\mathbf{d}$ de l'orientation des molécules sur le plan xOy. On a donc, après le passage dans la cellule, un déphasage $\delta_{\mathrm{N}}=2 \pi \mathrm{h}(\mathrm{r}) \Delta \mathrm{n} / \lambda$ entre ces deux types de

\section{Complément terminologique}

\section{sur les cristaux liquides nématiques}

L'article de Pawel Pieranski illustre le débat autour de la création en science de mots nouveaux en français, dont les Reflets de la physique ( ${ }^{\circ} 46$, p. 46 , et $n^{\circ} 49$, p. 41 ) se sont fait l'écho, débat lié à la question de l'usage du français en sciences aujourd'hui, alors que les découvertes sont d'abord publiées en langue anglaise.

L'effort de terminologie et de néologie a été très important dans le domaine de la matière molle, et en particulier des cristaux liquides, depuis les travaux de Georges Friedel et Charles Mauguin dans les années 1930. Il s'est traduit par la création de mots nouveaux tels que smectiques, mésophases, gouttes à gradins, structure en chevrons... qui sont passés dans la langue internationale et ont contribué à rendre compte d'une activité très féconde en France. Cet effort s'est prolongé dans les travaux de Pierre-Gilles de Gennes, avec de nombreux emprunts au vocabulaire classique du français (éponges, pincements, cloques, reptation...).

Pawel Pieranski nous en donne plusieurs exemples aujourd'hui avec, notamment, le mot " cunéitropisme ». Cette volonté accompagne alors une recherche où l'image est particulièrement riche et essentielle pour la compréhension : le mot prolonge l'image. Mais elle identifie aussi le créateur de la recherche. Cette pratique peut-elle être généralisée ?

Étienne Guyon $(*)$ et Danielle Candel $(* *)$

(*) Laboratoire PMMH, ENS Paris

(**) Histoire des théories linguistiques, Université Paris 7

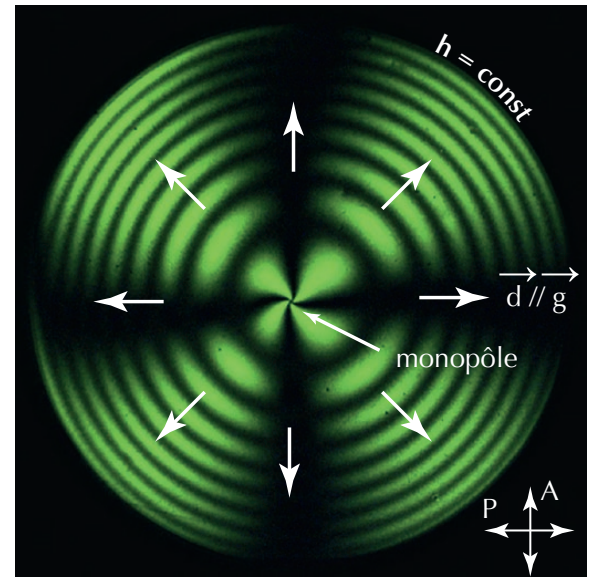

2. Effet du cunéitropisme : orientation de la texture pseudo-planaire par le gradient d'épaisseur. Cette image de la texture pseudo-planaire est similaire à celle de la figure 1f, mais ici elle est obtenue en lumière polarisée monochromatique. Les franges d'interférence circulaires appelées isochromes sont les lignes d'égale épaisseur h. Les quatre franges radiales appelées isogyres indiquent que la texture pseudoplanaire est disposée radialement. La projection $\mathbf{d}$ de I'orientation des molécules sur le plan x0y est donc parallèle au gradient d'épaisseur (d // g).

rayons ( $r$ est la distance à l'axe). Les isochromes noires correspondent donc aux valeurs de $r$ pour lesquelles $\delta_{N}=(2 N+1) \pi$ $(\mathrm{N}=0,1,2 \ldots)$. $\mathrm{A}$ biréfringence $\Delta \mathrm{n}$ constante, ces isochromes noires sont donc bien des franges circulaires sur lesquelles $\mathrm{r}$ et $\mathrm{h}$ sont constants. Le gradient d'épaisseur $\mathbf{g}=\nabla$ h, qui leur est perpendiculaire, est, lui, orienté radialement et dirigé vers l'extérieur (fig. 1f et fig. 2).

Les quatre autres franges noires sont radiales et forment une croix. Appelées isogyres (traduction de "même rotation "), ces franges marquent les points où le vecteur $\mathbf{d}$ caractérisant l'orientation azimutale des molécules est parallèle ou perpendiculaire à la polarisation imposée par P (fig. 1f et fig. 2). L'expérience montre d'ailleurs bien que, quand on fait tourner de concert les polariseurs $\mathrm{A}$ et $\mathrm{P}$, ces isogyres suivent leur rotation.

\section{Modèle analogique du cunéitropisme}

On peut comprendre simplement le cunéitropisme à l'aide d'une expérience analogique modélisant la structure pseudoplanaire locale entre une lentille et un plan : pour simplifier, la lentille est représentée 

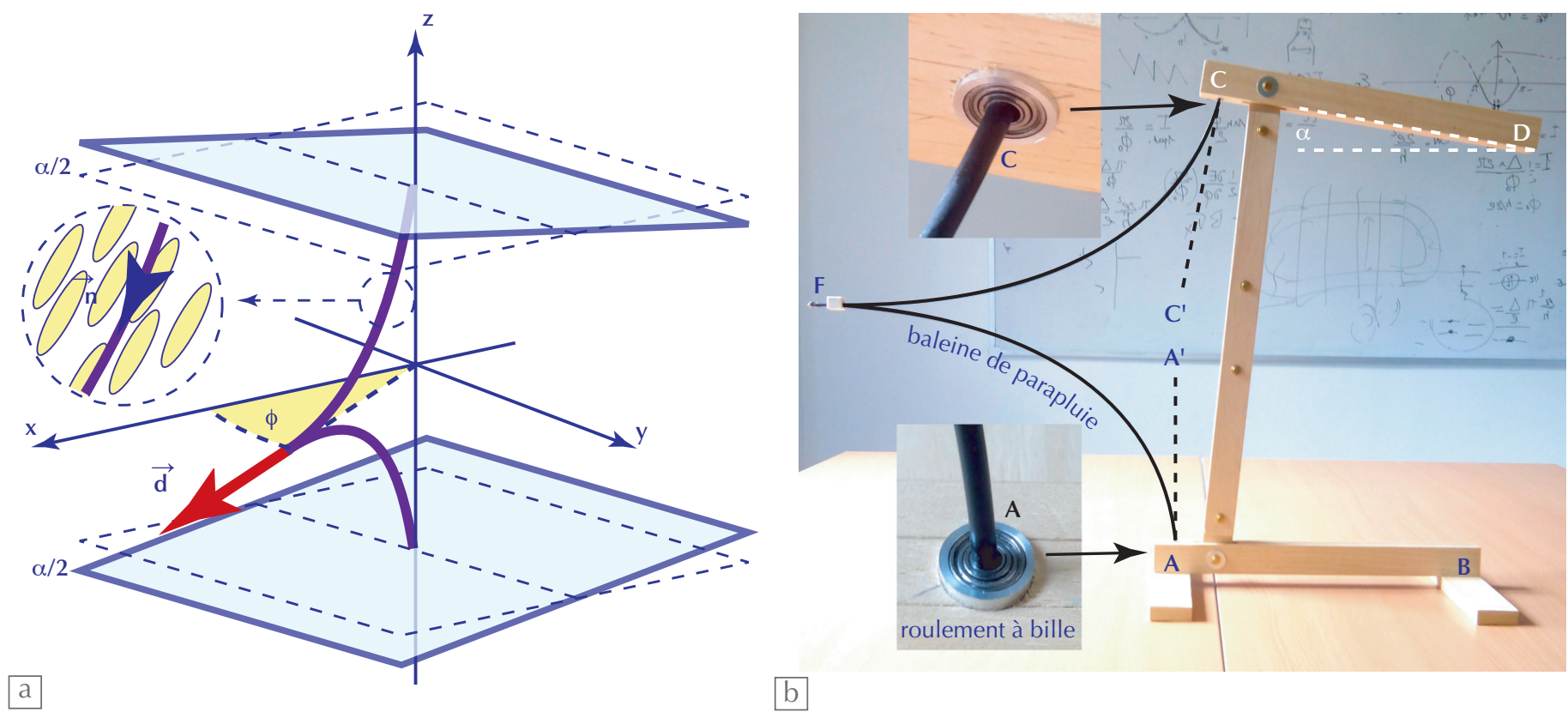

$\mathrm{b}$

3. Modélisation du cunéitropisme de la texture pseudo-planaire.

(a) Vue de la structure pseudo-planaire d'un nématique entre deux plans formant un angle $\alpha$ et modélisant localement l'espace entre une lentille et un plan ; les axes $x$ et y correspondent respectivement aux directions radiale et orthoradiale pour la lentille, et l'axe z est normal au plan médian au point considéré. Courbes bleu foncé : lignes de champ du directeur $\mathbf{n}$ situées dans un plan contenant l'axe $z$ et formant un angle $\phi$ avec $x 0 z$. Ces lignes de champ aboutissent aux intersections de l'axe $z$ avec les deux plans. (b) Modèle analogique mécanique. $A B C D=$ cadre de support. $\alpha=$ angle ajustable entre $A B$ et $C D$. $A F C$ : baleines de parapluie tangentes en leur point de jonction $F$ et maintenues perpendiculaires à $A B$ et $C D$ en leur point d'attache par des roulements à billes qui leur permettent de tourner ( $\phi=$ angle entre $A F C$ et le plan du cadre $A B C D)$.

\section{$\gg>$}

par le plan tangent au point considéré, qui forme un angle $\alpha$ avec l'autre plan (fig. 3a). Le modèle mécanique (fig. 3b) est formé de deux baleines de parapluie (ligne CFA sur la figure 3b) représentant les deux lignes de champ du directeur $\mathbf{n}$ montrées dans la figure $3 \mathrm{a}$ : leur élasticité simule celle du directeur $\mathbf{n}$, tandis que la tangente locale correspond à son orientation. Deux extrémités des baleines sont tangentes à leur point de jonction F, et elles sont perpendiculaires à leurs deux autres extrémités aux côtés $\mathrm{AB}$ et $\mathrm{CD}$ d'un cadre support représentant les deux plans de la figure $3 \mathrm{a}$; on reproduit ainsi l'ancrage homéotrope du cristal liquide, et l'angle $\phi$ entre ACF et le plan du cadre (équivalent à l'angle $\phi$ du schéma 3a) reste libre de varier (voir légende de la figure $3 b$ ).

En faisant varier l'angle $\phi$, on constate que la position la plus favorable énergétiquement correspond à $\phi=0$; par ailleurs, la force de rappel lorsqu'on s'en écarte augmente lorsqu'on fait varier $\alpha$ en déformant le cadre. Si l'angle $\alpha$ était 0 , la rotation de la " fourche " AFC serait libre, car l'énergie de déformation élastique des baleines, qui est de l'ordre de $F_{\text {él }}=(K / 2)\left(\pi^{2} / h\right)$, serait indépendante de son orientation azimutale.
En revanche, lorsque l'angle $\alpha$ n'est pas nul, il faut remplacer $\pi$ par $\pi-\alpha \cos \phi$ dans l'expression de $F_{\text {él }}$ et on obtient au premier ordre en $\alpha$ :

$$
\mathrm{F}_{\text {él }}=(\mathrm{K} / 2)\left(\pi^{2} / \mathrm{h}\right)-\mathrm{K}(\alpha \pi / \mathrm{h}) \cos \phi .
$$

Le minimum de cette énergie élastique est alors obtenu pour $\phi=N \star 2 \pi(N=0,1,2 \ldots)$.

Pour la texture pseudo-planaire de la figure 3a, le minimum d'énergie libre est obtenu pour ces mêmes valeurs de $\phi$ : d est alors parallèle au gradient d'épaisseur g et c'est donc celui-ci qui lève la dégénérescence azimutale de la texture. Dans un échantillon formé entre une lentille sphérique et une lame plane, l'axe x est remplacé par la direction radiale : on favorise donc la texture pseudo-planaire orientée radialement. Les textures des figures $1 \mathrm{f}$ et 2 correspondent bien au minimum $\mathrm{N}=0$ du terme cunéitropique.

\section{Paroi de $2 \pi=$ soliton}

Nous venons de voir que, de façon générale, la texture pseudo-planaire radiale est favorisée pour les angles azimutaux multiples entiers de $2 \pi: \phi(r)=N 2 \pi$, $\mathrm{N}=0,1,2 \ldots$ Près du ménisque, la solution $\phi=0$ s'impose à cause de l'ancrage homéotrope à l'interface nématique/air (n perpendiculaire à cet interface); par contre, à une distance suffisante du ménisque, on peut imposer un autre choix, par exemple $\mathrm{N}=1$.

Pour ce faire, on soumet la texture radiale homogène, comme celle des figures $1 \mathrm{e}, 1 \mathrm{f}$ et 2 , à un champ magnétique horizontal suffisamment fort, tournant lentement dans le plan de l'échantillon. L'expérience montre [6] qu'après un tour complet du champ, suivi de sa suppression et d'une relaxation d'une minute, on voit apparaitre dans la texture pseudo-planaire une "paroi " circulaire (fig. 4a) : cette paroi contient quatre isogyres sombres correspondant chacune à des orientations constantes de la composante azimutale $\mathbf{d}$ de $\mathbf{n}$ et espacées de $\pi / 2$. En dehors de la paroi, les isogyres se raccordent à des lignes radiales disposées à $90^{\circ}$ les unes des autres et situées au milieu des zones sombres. Chacune de ces isogyres est marquée par une ligne continue d'une couleur différente dans la figure 4a et les orientations correspondantes de $\mathbf{d}$ sont indiquées par des flèches blanches dans la figure $4 b$, qui est l'agrandissement du petit rectangle en tirets dans l'image 4a. 

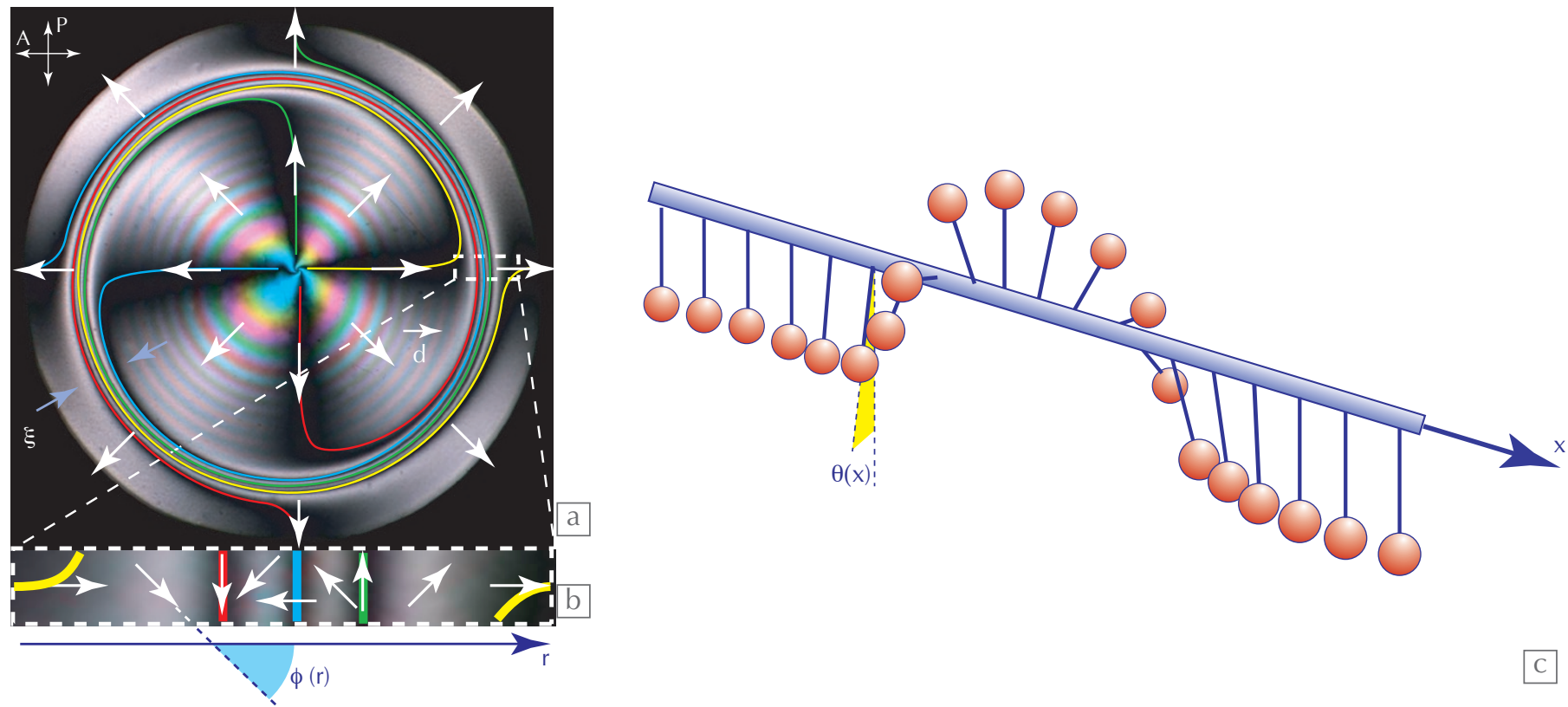

4. Paroi de $2 \pi$ d'une texture pseudo-planaire et analogie avec un soliton.

(a) Vue entre polariseur et analyseur croisés (flèches en haut et à gauche) d'une texture pseudo-planaire contenant une paroi de $2 \pi$ circulaire. Traits colorés : lignes isogyres correspondant à des angles de la composante horizontale $\mathbf{d}$ du directeur avec le polariseur égaux à $0, \pi / 2$, $\pi$ et $3 \pi / 2$. La largeur $\xi$ de la paroi est indiquée par des flèches bleues.

(b) Agrandissement de la partie de la figure (a) à l'intérieur du rectangle en tirets à droite. Les codes de couleur sont les mêmes que dans la figure (a).

(c) Analogue mécanique des parois de $2 \pi$ : soliton sur une chaine de pendules couplés par une tige élastique, sur laquelle ils sont fixés rigidement et dont l'énergie gravitationnelle $-m g L \cos [\theta(x)]$ est analogue au terme cunéitropique de la texture pseudo-planaire $-K(\alpha / h) \cos [\phi(r)]$.

Nous savons que les isogyres noires sont les lieux géométriques où le vecteur $\mathrm{d}$ est parallèle ou perpendiculaire à la polarisation imposée par $\mathrm{P}$; à partir des figures $4 \mathrm{a}$ et $4 \mathrm{~b}$ et en utilisant cette règle, nous allons démontrer que $\mathbf{d}$ tourne de $2 \pi$ lorsqu'on traverse la paroi. Commençons par la plage noire à gauche de l'image $4 b$, juste avant la paroi et marquée par le trait jaune : l'orientation du vecteur $\mathbf{d}$ (flèche blanche à gauche) y est celle de l'axe r (fig. 4a). Au milieu de la plage noire suivante (trait rouge dans la figure $4 \mathrm{~b}$ ), le vecteur $\mathbf{d}$ a tourné de $\pi / 2$ (flèche blanche). Entre les deux, le vecteur $\mathbf{d}$ tourne continûment de 0 à $\pi / 2$, en passant par $\pi / 4$ au milieu de la première zone claire de la paroi (flèche blanche dans la figure 4b). En continuant de cette façon, on trouve que l'angle $\phi$ varie au total de $2 \pi$ quand on traverse la paroi et on retrouve une autre ligne jaune correspondant à la même orientation (à $2 \pi$ près).

Il nous reste à comprendre quel mécanisme détermine la largeur $\xi$ de la paroi (fig. 4a). Nous allons le faire au moyen d'un autre modèle mécanique : une file de pendules de masse $m$ et de longueur $L$ représentée sur la figure $4 c$. Ce modèle est une représen- tation pédagogique classique d'un soliton, supposé ici statique. L'angle des pendules avec la verticale est appelé $\theta$ et est équivalent à l'angle $\phi$ entre $\mathbf{d}$ et le gradient d'épaisseur. L'état de plus faible énergie (équivalent du cas $\mathrm{N}=0$ ) correspond à une orientation verticale vers le bas de tous les pendules. Si on fait tourner de $2 \pi$ une des extrémités de la chaîne de pendules (et si la tige de couplage n'est pas trop rigide), on obtiendra la configuration de la figure $4 c$ qui est l'équivalent du cas $\mathrm{N}=1$ et subsiste même si on arrête d'agir sur les pendules. L'énergie globale du système associe la variation d'énergie potentielle de pesanteur des masses par rapport à leur position de base $\theta=0: \mathrm{E}_{\mathrm{g}}=-\mathrm{mgL} \cos [\theta(\mathrm{x})]$, et l'énergie élastique associée à la torsion de la tige. Cette torsion est proportionnelle à $(\Delta \theta / \Delta x)^{2}$, où $\Delta \theta$ est l'angle entre deux pendules voisins et $\Delta \mathrm{x}$ la distance qui les sépare. Ces deux termes sont respectivement analogues au terme cunéitropique $\mathrm{F}_{\mathrm{c}}=-\mathrm{K}(\alpha / \mathrm{h}) \cos [\phi(\mathrm{x})]$ et à un terme proportionnel à $(\mathrm{d} \phi / \mathrm{dr})^{2}$ dans la texture pseudo-planaire ; ce dernier terme correspond à l'énergie élastique associée au gradient radial de l'orientation du champ $\mathbf{d}$. Dans ce modèle mécanique, la variation de $\theta$ avec la distance $\mathrm{x}$ et, donc, la largeur suivant $\mathrm{x}$ de la zone perturbée (équivalente à la largeur de paroi $\xi$ qui est toujours supposée $<<\mathrm{R}$ ) sont obtenues en minimisant l'énergie globale.

Nous obtenons de même l'expression de l'énergie de la paroi intégrée sur $\mathbf{r}$ : $\mathrm{F}_{\text {tot }} \approx \int\left[\left(\mathrm{K}^{\prime} / 2\right) \mathrm{h}(\mathrm{d} \phi / \mathrm{dr})^{2}-\mathrm{K}(\alpha \pi / \mathrm{h}) \cos \phi\right] \mathrm{dr}$ (ici, K' est une constante élastique). La minimisation de cette fonctionnelle conduit à l'équation de sine-Gordon, omniprésente dans la théorie des solitons :

$$
\mathrm{d}^{2} \phi / \mathrm{dr}^{2}-\left(1 / \xi^{2}\right) \sin \phi=0,
$$

où $\xi=h\left[\left(K^{\prime} / K\right)(1 / \pi \alpha)\right]^{1 / 2}$. La largeur de la paroi dépend donc à la fois des valeurs locales de l'épaisseur $\mathrm{h}(\mathrm{R})$ de la cellule et de l'angle $\alpha(\mathrm{R})$ : celles-ci sont facilement prédites pour toutes les valeurs de $\mathrm{R}$ à partir du rayon de courbure de la lentille et de sa distance au plan. L'énergie totale de la paroi est proportionnelle à sa longueur $2 \pi \mathrm{R}$ : le rayon $\mathrm{R}$ diminue donc au cours $\mathrm{du}$ temps pour réduire cette énergie et devient nul au bout d'une vingtaine d'heures.

Nous avons pu vérifier expérimentalement, en mesurant la variation de $\xi$ avec R, que la valeur de $\xi$ correspondait bien à l'expression ci-dessus [6]. 


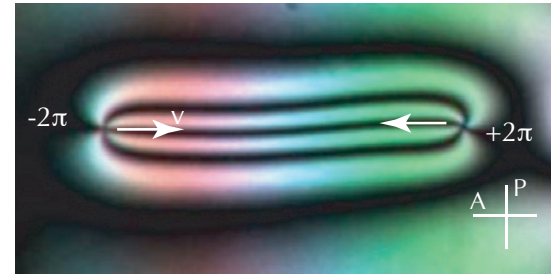

a

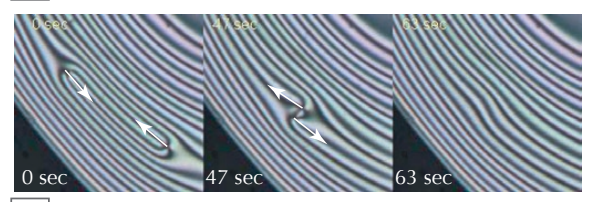

e

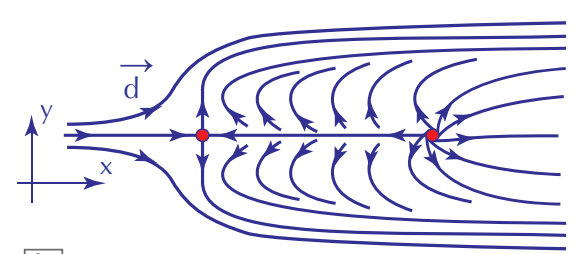

b

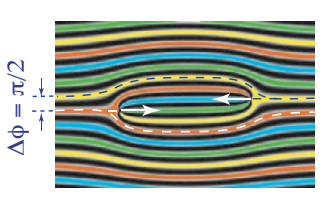

f
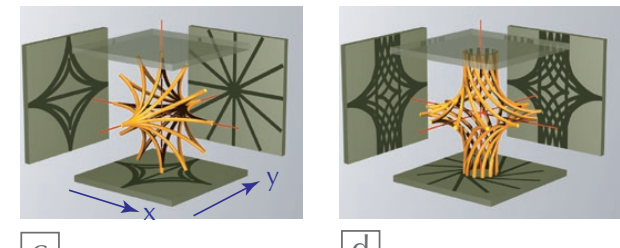

d

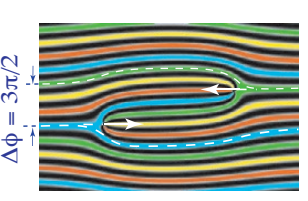

$g$

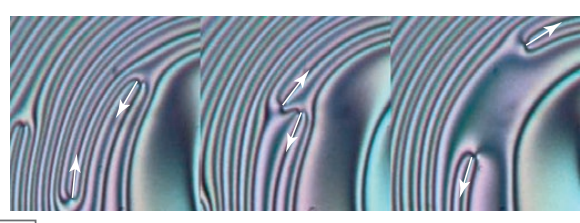

$\mathrm{h}$

5. Estimation de la section efficace de l'annihilation des paires monopôle-antimonopôle nématiques.

(a) Vue entre polariseurs croisés d'une paire monopôle-antimonopôle créée dans une structure pseudo-planaire. Les deux défauts sont liés par une paroi de $2 \pi$ équivalente à celle de la figure 4. (b) Schéma du champ $\mathbf{d}$ déduit de la photo (a). (c) et (d) Structures tridimensionnelles de l'antimonopôle (c) et du monopôle (d). (e) Collision et annihilation d'une paire monopôle-antimonopôle en mouvement. (f) - (g) Structure des isogyres autour d'une paire monopôle-antimonopôle pour deux distances différentes entre les trajectoires. (h) Collision d'une paire sans annihilation. Dans les images $(\mathrm{a}),(\mathrm{e}),(\mathrm{f}),(\mathrm{g})$ et $(\mathrm{h})$, les flèches blanches représentent la vitesse de déplacement des monopôles.

\〉

\section{Annihilation des paires monopôle-antimonopôle}

Des manipulations appropriées de la texture pseudo-planaire au moyen d'un champ magnétique et d'un écoulement permettent de créer de façon contrôlée des paires de défauts ponctuels telles que celle de la figure $5 \mathrm{a}$ [5]. L'analyse de cette image permet de comprendre que les structures tridimensionnelles des deux défauts peuvent être obtenues par des déformations continues à partir de celle du monopôle hyperbolique de la figure E2b. Le défaut noté « $-2 \pi$ » sur la figure 5 a et dont la structure est montrée sur la figure $5 c$, n'est rien d'autre que ce monopôle tourné de $\pi / 2$ autour de l'axe $\mathrm{x}$; la structure du défaut noté « $+2 \pi$ » (fig. $5 \mathrm{~d}$ ) s'en déduit, elle, par la rotation locale du champ du directeur autour de l'axe $z$.

Sur la figure $5 a$, les deux défauts sont liés par une paroi de $2 \pi$ (schématisée sur la figure $5 b$ ) similaire à celle de la figure 4 . Cette paroi tend à réduire sa longueur, ce qui attire les défauts l'un vers l'autre de sorte que leur collision est inévitable.

Nous avons étudié de telles collisions dans des structures (fig. 5e-f-g) obtenues en faisant tourner d'un grand nombre de tours le champ magnétique qui crée les parois (au lieu d'un seul tour dans la figure 4). On obtient alors un feuilletage de nombreuses parois juxtaposées, toutes perpendiculaires au gradient $\mathbf{g}$ de la phase $\phi$. Ces structures planaires contiennent des défauts du même type que ceux de la figure $5 \mathrm{a}$ : on voit ainsi sur la figure 5 e une paire de défauts $(2 \pi$ et $-2 \pi)$ qui se déplacent en sens inverse parallèlement aux parois (et donc perpendiculairement à g). Dans la figure $5 \mathrm{e}$, on a une collision des deux défauts qui entraine une annihilation de la paire (dernière image), mais cette issue fatale n'est pas inéluctable : le résultat de la collision dépend de la distance entre les trajectoires, comme on peut le comprendre d'après les vues $5 \mathrm{~g}$ et $5 \mathrm{~h}$ qui montrent les trajectoires des différentes isogyres. Ainsi, dans la configuration de la figure $5 \mathrm{~g}$, où la distance est plus grande que dans le cas $5 f$ correspondant à l'annihilation, les deux monopôles de la paire se rencontrent sans s'annihiler.

En répétant l'expérience, on arrive à la conclusion que l'annihilation a lieu lorsque la différence de phase $\Delta \phi$ entre les trajectoires est inférieure à $\pi$, alors qu'elles se croisent simplement si $\Delta \phi>\pi$. Comme la distance transverse qui correspond à une variation de phase de $\pi$ est égale à $\xi / 2$, on peut alors définir une "section efficace " de collision $\rho=\xi / 2$ : c'est la limite inférieure de la distance entre trajectoires au-delà de laquelle on n'a pas d'annihilation (cette section efficace a la dimension d'une longueur, car on a affaire à une structure

\section{Références}

1• M. Kleman et 0. D. Lavrentovich, Soft matter physics, Springer (2003).

2- P. Oswald et P. Pieranski, Nematic and Cholesteric Liquid Crystals, Taylor \& Francis (2005), et Smectic and columnar Liquid Crystals, Taylor \& Francis (2006).

3• M. Kléman et 0. D. Lavrentovich, Phil. Mag., 86 (2006) 4117-4137. bidimensionnelle). De plus, $\rho$ est reliée à la vitesse $\mathrm{v}$ de propagation des monopôles par $\rho=A \pi / v$ (A est une constante) : on observe, en effet, expérimentalement que $\mathrm{v}$ est proportionnelle au gradient local de la phase, avec $\mathrm{v}=\mathrm{A} \mathrm{d} \phi / \mathrm{dr}=2 \pi \mathrm{A} / \xi$. La longueur d'onde $\xi$ de la texture pseudoplanaire enroulée joue alors un rôle analogue à celui de la longueur d'onde de de Broglie $\boldsymbol{\lambda}=\mathrm{h} /(\mathrm{mv})$ pendant les collisions de neutrons lents avec des noyaux atomiques [6].

Sans être cités explicitement, les noms de Jacques Friedel et Pierre-Gilles de Gennes apparaissent en filigrane sur toutes les pages de cet article. Au Laboratoire de Physique des Solides d'Orsay comme dans bien d'autres lieux, ils ont su favoriser un dialogue fructueux entre les disciplines de la matière condensée. Les concepts de défauts topologiques représentent des éléments importants du langage unificateur de ce dialogue.

\section{Remerciements}

Ce travail est le résultat d'une collaboration avec les équipes portugaise (M.H. Godinho et al.) et slovène (S. Copar et al.), ainsi qu'avec M. Dazza, stagiaire M2 de l'ENS de Cachan. L'auteur remercie P. Oswald pour les discussions sur le cunéitropisme et pour le rappel du travail précurseur de J. M. Gilli et al. sur la texture pseudo-planaire et ses défauts [4]. La mise en forme de l'article doit beaucoup aux remarques éclairées des rapporteurs, ainsi qu'au concours généreux de C. de Novion, J.P. Hulin et M. Leduc, que je tiens à remercier très chaleureusement. Enfin, la rédaction de l'article a été initiée par E. Guyon, auquel l'auteur est particulièrement reconnaissant.

\footnotetext{
4• J.M. Gilli et al., Liquid Crystals 23 (1997) 619-628.

5• P. Pieranski, M.H. Godinho et S. Copar, "Persistent quasiplanar texture: Its properties and topological defects", Phys. Rev. E 94 (2016) 042706.

6• H.A. Bethe, "Theory of disintegration of nuclei by neutrons", Phys. Rev. 47 (1935) 747-759.
} 